\title{
A computational method for pressure wave machinery to internal combustion engines and gas turbines
}

\author{
A. Fatsis ${ }^{1}$, M. Gr. Vrachopoulos ${ }^{1}$, S. Mavrommatis ${ }^{1}$, \\ A. Panoutsopoulou ${ }^{2} \&$ F. Layrenti ${ }^{1}$ \\ ${ }^{I}$ Technological University of Chalkis, \\ Department of Mechanical Engineering, Greece \\ ${ }^{2}$ Hellenic Defense Systems S.A., Greece
}

\begin{abstract}
Pressure wave machinery constitutes promising devices in various engineering propulsion applications such as superchargers in automobile engines and topping devices in gas turbines. This article presents applications of a numerical model for the flow field prediction inside wave rotors. The numerical method used consists of an approximate Roe solver that takes into account viscous and thermal losses inside the rotor as well as leakage losses at the extremities of the rotor. The model is extensively validated and then is applied on configurations suited for automobile engine supercharging and for topping devices for gas turbines. For both cases, satisfactory results are obtained by the comparison of the numerical predictions against experimental data available in the literature. It is concluded that the present method can accurately predict the basic unsteady flow patterns inside the rotor.

Keywords: pressure wave supercharger, wave rotor, internal combustion engine, gas turbine, unsteady flow.
\end{abstract}

\section{Introduction}

Pressure wave superchargers or wave rotors or Comprex ${ }^{\circledR}$ are rotating devices in which energy is transferred between two gaseous fluids by short time direct contact of the fluids in slender flow channels. They are composed of two concentric cylinders between which, radial straight planes are arranged giving 
rise to long channels of constant cross section, Berchtold [1]. Lateral nonrotating perforated flanges (stators) are mounted upstream and downstream the rotor. Through the openings of the stators that are commonly called inlet and outlet ports, the air and the hot gases enter and exit the rotor.

Different applications of wave rotors can be obtained depending on the following parameters: (i) the number of ports upstream and downstream the rotor, (ii) the dimensions of the ports, (iii) the aero-thermodynamic quantities specified at the inlet and outlet of the ports and (iv) the direction of the inflow and outflow at each port. Initially wave rotors were designed to supercharge internal combustion engines. In such applications, the ports are connecting the rotor to the fresh air intake, to the exhaust pipe and to the inlet and outlet of the combustion chamber of the engine. Automobile applications of the pressure wave supercharger over a wide range of car and truck diesel engines, showed a fast response to changes in the engine load, resulting in almost instantaneous availability of maximum torque, according to Mayer et al. [2].

Wilson and Fronek [3], present the pressure divider, that is another application of wave rotors. This type of wave rotor is generally equipped with three-ports. It is used to split the inlet flow in two flows; one at a higher pressure and the other at a lower one. Fatsis [4], examined the benefits of wave rotors as topping devices when applied to different types of gas turbines for aeronautical applications.

The flow inside the rotor is unsteady. It is dominated by propagation of compression and expansion waves that interact with each other and reflect on the solid walls of the upstream and downstream stators as well as on the inflow and on the outflow boundaries. One-dimensional methods based on the Euler equations are mainly used including modelling for losses that occur inside the rotor e.g. Fatsis et al. [5], Paxson [6]. Two-dimensional methods such as the one of Welch [7] are rather time-consuming and are mainly suited to study in detail specific regions of interest of the flow field inside the rotor, such as finite opening / closing effects and shock wave - boundary layer interaction.

The present contribution aims to present a general and accurate numerical tool suited for the analysis of unsteady flows encountered inside different types of wave rotors. After a brief description of the numerical method, the model is validated against experimental data for the flow inside a three-port pressure divider. Then the model is implemented to predict the flow inside a reverse flow wave rotor that can be used as a supercharger of internal combustion engines. Comparisons with measurements show that the present method gives accurate results for the cases examined and that it is able to capture the basic unsteady flow phenomena inside the rotor.

\section{Numerical method}

\subsection{Assumptions}

In the existing pressure wave superchargers manufactured so far it is observed that the length of each wave rotor channel is much larger than its height and 
width, according to Mayer et al. [2]. This justifies the approximation of the flow inside a channel formed by two consecutive blades with the flow inside a shock tube. This consideration simplifies the real three-dimensional and unsteady flow inside the rotor channels with a one-dimensional unsteady flow inside a shock tube. A two-dimensional analysis method, though, could clarify the limits of applicability of one-dimensional methods. As a first approximation, this approach can be considered realistic. Nevertheless pressure losses; viscous phenomena and leakage existing in the flow inside a real wave rotor are taken into account in the present method, Fatsis et al. [5].

\subsection{Spatial discretisation}

Experience using different numerical schemes showed that upwind schemes are well suited for compressible flows including discontinuities such as shock waves, Hirsch [8]. The second order accurate scheme of Roe [9] was chosen for the space discretisation of the system of the partial differential equations, Fatsis et al. [5].

\subsection{Time integration}

Since the behaviour of the unsteady flow inside the wave rotor channels is of interest in the present study, the time integration scheme should offer a high accuracy. For this reason, the four-step Runge-Kutta scheme consists of an attractive choice, Fatsis et al. [5]. Studies show that the above scheme is second order accurate for non-linear equations, Hirsch [8].

\subsection{Boundary conditions}

During the operation of a wave rotor it is possible to have any type of the following boundary conditions: subsonic or supersonic towards the inlet and outlet of the computational domain (rotor channel). According to the theory of characteristics given by Hirsch [8] the numerical model employs different types of boundary conditions depending on the nature of the flow in the extremities of the wave rotor channels.

All boundary conditions implemented use the compatibility equations method described by Fatsis et al. [5]. The boundary conditions are of reflecting type, simulating infinite reservoirs with constant flow conditions connected to the rotor inflow and outflow ports. Under these boundary conditions when moving shock waves reach the rotor extremities, reflect back to the rotor channel interacting with other.

\section{Validation}

The model has been validated first on shock tube flow by Fatsis et al. [5], where analytical solutions can be found by Kentfield [10]. A second validation test that will be presented here refers to the calculation of the flow inside the three-port through-flow wave rotor or "pressure divider". This wave rotor was 
manufactured and instrumented to validate numerical models aiming to analyse its flow pattern. The experimental data of the unsteady pressure distribution at the following axial positions of the rotor, $x / L=0.025,0.50,0.975$, were reported by Wilson and Fronek [3].
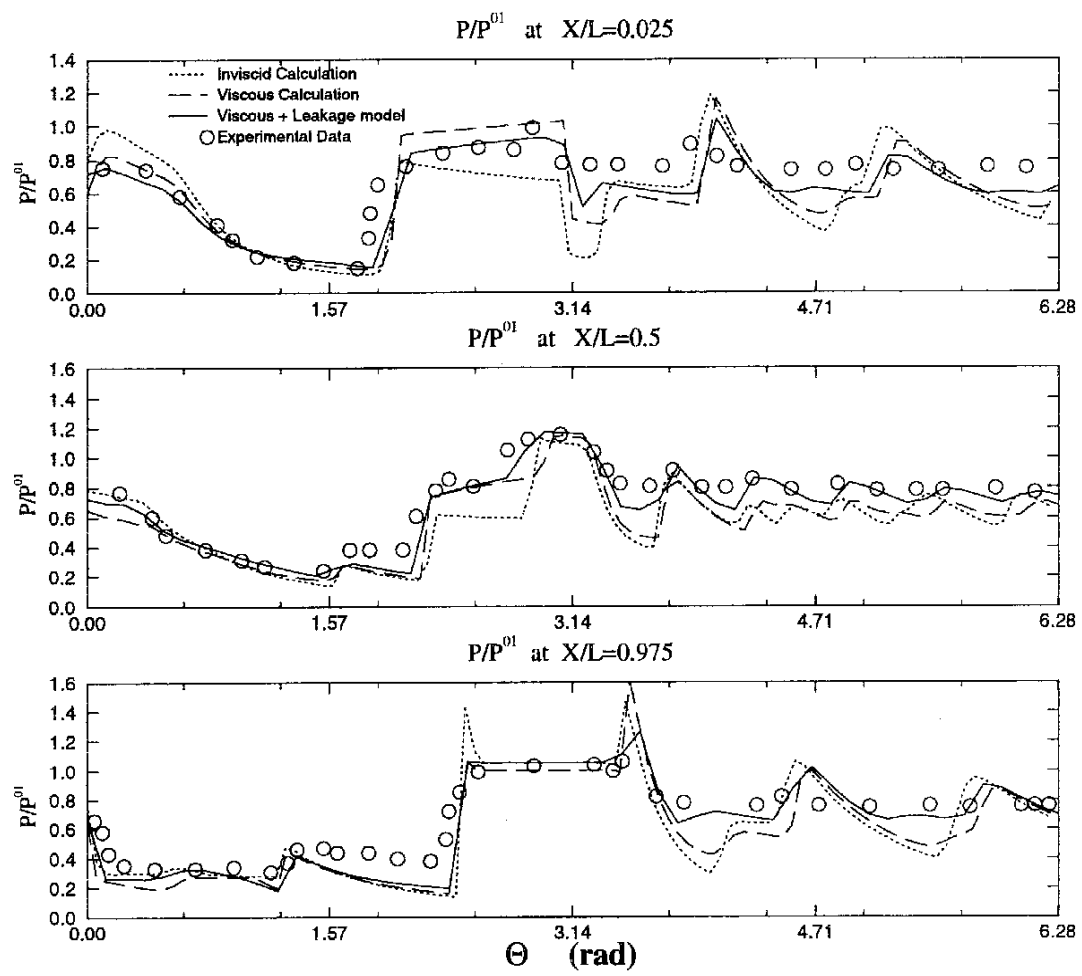

Figure 1: Comparison between numerical predictions and experimental data for the three-port wave rotor.

Figure 1 presents the comparison between the predictions obtained using the present numerical model and experimental data from Wilson and Fronek [3] illustrated by circles. Dotted lines present inviscid flow calculations, long dashed lines present results obtained including viscous and thermal losses and continuous lines present the predictions of the final model which also includes leakage losses at the extremities of the rotor (improved model). Inviscid flow predictions show larger amplitude of oscillation for the three axial locations. This behaviour is expected because upwind schemes (such the one used in this model) include just the minimum numerical damping provided by the numerical flux limiters, Roe [9]. Predictions including viscous and thermal losses give better results than the inviscid flow predictions when compared to the experimental data. The amplitude of pressure oscillations of the model including viscous and thermal losses is reduced due to the dissipation resulting from the 
addition of the loss modelling. Ultimately, results obtained adding also leakage source terms, improve further the numerical predictions. Although leakage source terms are included only at the first and last computational cells, they affect the flow field even at $\mathrm{x} / \mathrm{L}=0.5$. One can observe that the agreement between the final (improved) model and experimental data is very satisfactory for the three axial locations examined. There are some regions, though, like the one corresponding to the opening of the inflow port at left $(x / L=0.025$, $\theta \in[2,3] \mathrm{rad})$ where the agreement is not so good. These differences occur due to the fact that the present model supposes that the flow is accelerated instantaneously when a rotor channel arrives in front of an inflow port. In reality there are losses associated to the inflow and outflow phenomena (finite opening time effects), which are not taken into account in the present numerical model.

\section{Reverse flow wave rotor}

This type of wave rotor is currently used in automobile applications, and experimental data on the subject has been published by Shreeve and Mathur [11] using the same experimental techniques as for the case of the three-port wave rotor described in the previous chapter. Figure 2(a), (b), shows predicted total pressure and total temperature inside the rotor, non-dimensionalised by the total pressure and temperature at the inlet of port (1), during one complete rotation. The rotor is supposed to rotate from the lower to the upper part of figure 2(a), (b). The abscissa is the non-dimensional distance $x / L$ extending from 0 to 1 and the ordinate corresponds to the circumferential coordinate $\Theta$ extending from 0 to $2 \pi$. The process in this cycle begins at the lower part of the figure 2(a), (b), when the port (4) towards the exhaust pipe opens and hot gases with a percentage of relatively cold, compressed air exit the rotor. As e result an expansion wave is generated that propagates to the left, hitting the upstream stator walls and then is reflected to the right as a compression wave. At this moment, port (1) opens and due to the large pressure difference, fresh air enters, scavenging the rotor cells and pushing the remaining gases towards the exhaust pipe port (4). As the port (4) closes, the compression wave that was initiated when port (1) was open hits the downstream stator wall and reflects to the left as a shock wave. This shock wave front is progressing faster than the speed of sound before it and it is simultaneously compressing and accelerating the air towards left. At that moment, port (2) opens and hot gases from the combustion chamber of the engine enter the rotor, creating a strong shock wave propagating to the left, compressing and pushing air and remaining gas towards the combustion chamber (port (3)), figure 2(a). When the front between the compressed air and hot gases reaches the port (3), this port closes, preventing the exhaust gases recirculation into the engine. Some of the air that has been contaminated remains in the rotor cells. As the port (3) towards the combustion chamber closes, a compression wave is created, pushing the remaining gas and compressed air towards the exhaust pipe port (4). This remaining air gives the 
energy to scavenge the cells, namely to replace the exhaust gas with air. From figure 2(b), one can see that mainly hot gases remain in the right part of the rotor, corresponding to ports (2) and (4), while the air remains in the left part of the rotor, corresponding to ports (1) and (3). Other researchers have made similar observations, e.g. Mayer et al. [2].

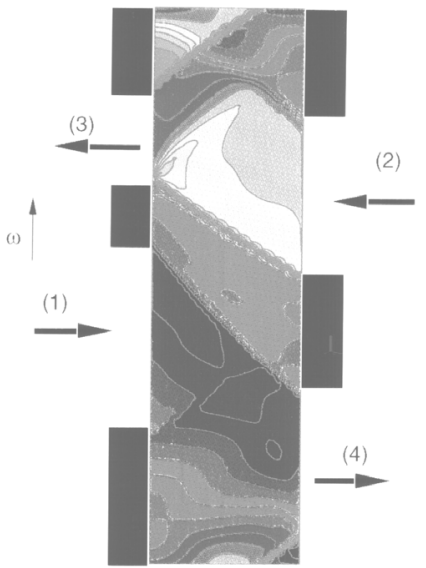

(a)

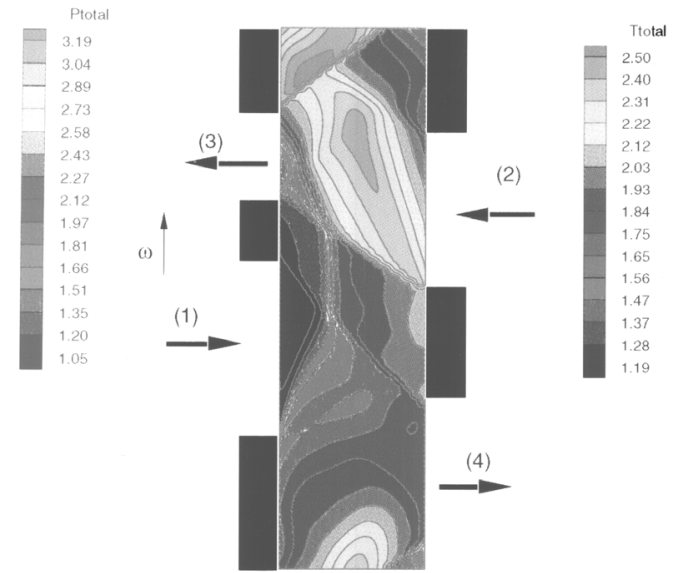

(b)

Figure 2: (a) Total pressure non-dimensionalised by the total pressure at port (1), (b) total temperature non-dimensionalised by the total temperature at port (1) field for the reverse flow four-port wave rotor. (1) Air at atmospheric conditions, (2) Compressed air from combustion chamber, (3) Hot gases to combustion chamber, (4) Hot gases and air to the exhaust.

The wave processes as described previously correspond to a "perfectly tuned" pressure wave supercharger according to the engine demands. In reality, a supercharger has to function over a wide engine operating range from very low up to very high rotating speeds. The pressure wave supercharger succeeded this wide range speed operation by using additional stator ports, called "pockets", which, however are not connected to any duct, Berchtold [1].

Detailed comparisons between predicted and measured static pressure distribution at axial locations (a) $x / L=0$ and (b) $x / L=1$ respectively are presented in figure 3. At $x / L=0$ predictions match the experimental data obtained from [19]. The measurement technique applied was similar to the one described in chapter 4. Small discrepancies occur at circumferential locations where $\theta \in$ $[\pi, 3 \pi / 2] \mathrm{rad}$, corresponding to port (1) and where $\theta \in[7 \pi / 4,2 \pi] \mathrm{rad}$ corresponding to the closing of port (3). At $x / L=1$ differences between experimental data and predictions occur at circumferential locations where $\theta \in[0, \pi / 4]$. rad, corresponding to the opening of port (4) and where $\theta \in[7 \pi / 4,2 \pi]$ rad corresponding to solid wall area. The comparison can be 
considered good taking into account the complexity of the unsteady flow inside the rotor channels and the fact that the experiment was carried on a real engine. Yet, incorporating loss model for the finite closing of ports effect, could improve the accuracy of numerical predictions in the circumferential positions corresponding to the closing of inflow / outflow ports.

(a) $X / L=0.0$

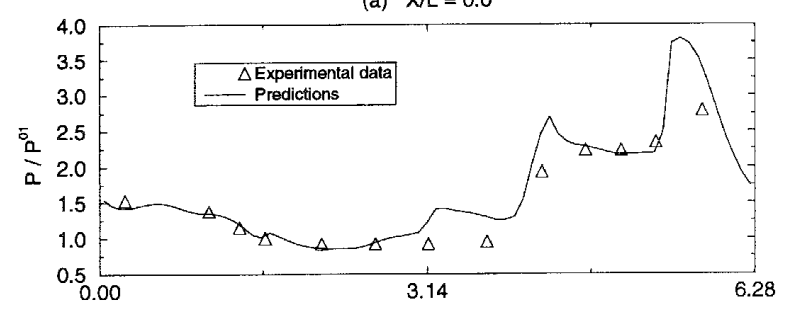

(b) $\mathrm{X} / \mathrm{L}=1.0$

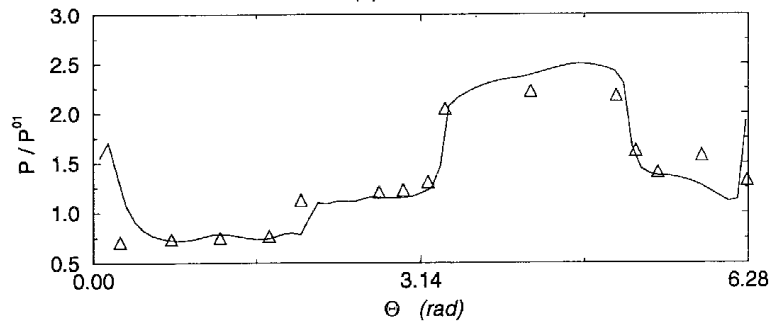

Figure 3: Comparison between computed and measured static pressure distributions for the reverse-flow wave rotor.

\section{Through-flow four-port wave rotor}

This type of four-port wave rotor is mainly suited for aeronautical applications due to its self-cooling capability, as it will be shown. Input geometrical data are published by Welch [7]. Figures $\backslash$ ref $\{$ fig:thru_p\} $\sim$ and $\backslash$ ref $\{$ fig:thru_t $\}$ show instantaneous total pressure and total temperature ruster plots for the throughflow wave rotor examined. For both figures, the abscissa is the non-dimensional $\mathrm{x} / \mathrm{L}$ distance extending from 0 to 1 and the ordinate corresponds to the circumferential coordinate $\theta$ extending from 0 to $2 \pi$.

The wave rotor is supposed to rotate from the lower to the upper part of the figure. Initially the rotor is filled with hot gases. The operation begins at the lower part where the hot gases expand when the port (4) towards the turbine opens, figure 4(a). The expansion fan which is created hits the left side walls and is reflected to the right. When it reaches the upper part of the port (4) towards the turbine, this port closes. A part of the expansion fan is reflected on the isopressure surface (forming thus a coalesced shock) and the rest is reflected on the solid wall. On the other hand, the port (1) from the compressor opens and relatively cold air enters the rotor. The contact interface between cold air and hot 
gases can be seen on figure 4(b). When the port (3) from the combustion chamber opens, a strong shock is created which interacts with the interface as it propagates to the right. At that moment, it is probable to anticipate extra losses due to the interaction, but Weber (1995) suggests that they can be neglected at a first approach. When the shock reaches the right end, it reflects on the walls and goes towards left. When the port (2) towards the combustion chamber opens, a part of hot gases (which did not leave the rotor when the port (4) towards the turbine was opened) goes out as well as the most of the compressed air included in the interface, figure 4(b). Then the port closes creating a left-propagating shock which interacts with the expansion fan produced when the port (3) from combustion chamber had been closed.

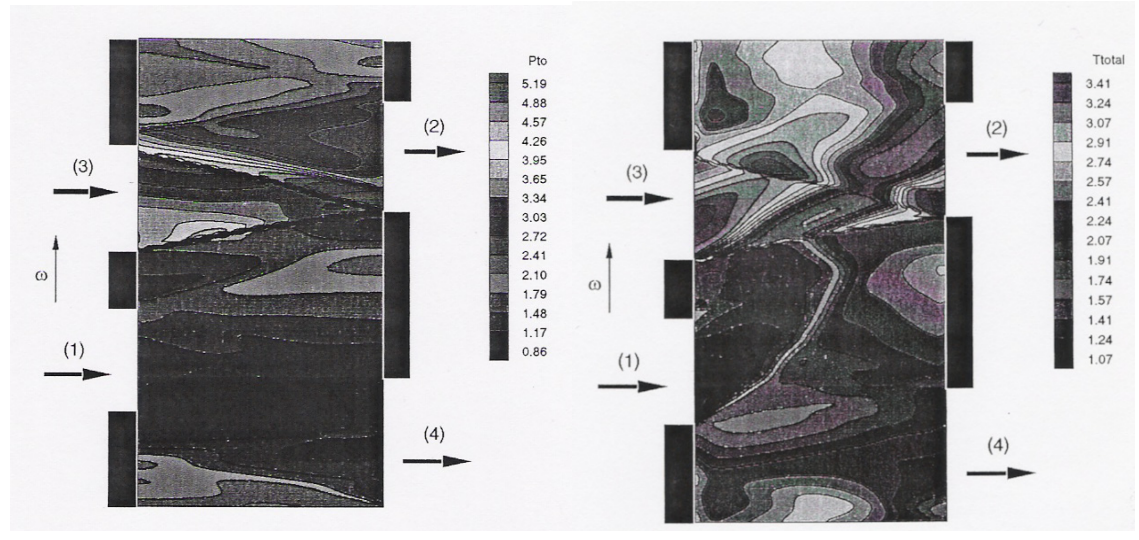

(a)

(b)

Figure 4: Instantaneous (a) total pressure and (b) total temperature contours for the four-port through-flow wave rotor. (1): From Compressor, (2): To Combustion Chamber, (3): From Combustion Chamber, (4): To Turbine.

Similar observations were done by Paxson [6] and Welch [7] on throughflow four-port cycle computations. From figure 4(a) one can see that the upper part (ports (2), (3)) corresponds to the high pressure part of the cycle, while the lower part (ports (1), (4)) corresponds to the low pressure part. The self-cooling aspect of the through-flow configuration can be clearly seen from figure 4(b) where cold air coming from compressor through the port (1) traverses the wave rotor channels and flows out towards the combustion chamber (port (2)). This allows the blade material to be cooled by forced convection and not only by conduction. One can also see the circumferential periodicity at the end of each rotation, as well as the fully unsteady character of the flow inside the rotor. The average total pressure at port (2) towards the combustion chamber is equal to 3.21, (Welch, [7] found from $2 D$ computations 3.15) fact which justifies the choice of $P R=3$ in the cycle analysis done in the beginning of this article. The 
wave rotor total pressure ratio of this simulation was $T_{4}^{0} / T_{1}^{0}=1.943$ and the total pressure ratio $P_{4}^{0} / P_{1}^{0}=1.145$. Welch [7] reports that $T_{4}^{0} / T_{1}^{0}=1.91$ and $P_{4}^{0} / P_{1}^{0}=1.12$ respectively. This means that the existing technology can provide the material for the wave rotor construction. From figures 4(a), (b) one can see that total pressure and temperature are not uniform at the port (4) towards the turbine. This creates extra losses in the ducts which were also taken into account in the cycle analysis.

\section{Conclusions}

A simple one-dimensional model, able to analyse unsteady flows inside multipleport wave rotors, was presented. A validation of the model was presented for a three-port wave rotor against experimental data available in the literature. Then the model was applied on a reverse-flow four-port pressure wave supercharger suited for internal combustion engines and for a through-flow wave rotor suited as a topping device for gas turbine performance enhancement. The numerical results obtained showed that the model is able to predict the unsteady flow field features inside the rotor channels, being in agreement with measurements and observations of other researchers. The self-cooling character of the through-flow wave rotor was verified from the fact that the mean blade temperature was found to be less than the turbine inlet temperature. Two-dimensional simulations in [11] can give an insight of the issue and may clarify the applicability limits of one-dimensional prediction methods. Including in the model the effect of pockets in the stator walls can lead to more realistic prediction of the performance map of pressure wave superchargers when integrated in internal combustion engines. That would give the possibility to study the behaviour of the wave processes inside the rotor at off-design conditions.

\section{Acknowledgment}

This publication was accomplished in the framework of Archimedes I-Support of Research Programs EПЕАEK II.

\section{References}

[1] Berchtold, M. Supercharging with Comprex. VKI Lecture Series 1982-01 entitled "Turbochargers and related problems", 1982.

[2] Mayer, A., Pauli, E., Gyrax, J. Comprex ${ }^{\circledR}$ Supercharging and Emissions Reduction in Vehicular Diesel Engines SAE Paper No. 900881, 1990.

[3] Wilson, J., Fronek, D. Initial Results from the NASA-Lewis Wave Rotor Experiment. AIAA Paper 93-2521, 1993. 
[4] Fatsis, A., Ribaud, Y. Thermodynamic analysis of gas turbines topped with wave rotors, Aerospace Science and Technology, 1999, No. 5, pp. 293-299.

[5] A. Fatsis, A. Lafond, Y. Ribaud, "Preliminary analysis of the flow inside a three-port wave rotor by means of a numerical model". Aerospace, Science and Technology, Vol. 2, No. 5, July 1998, pp. 289-300.

[6] Paxson, D.E. Comparison between Numerically modelled and Experimentally Measured Loss Mechanisms in Wave Rotors. Journal of Propulsion and Power, Vol.11, No.5, pp. 908-914, 1995.

[7] Welch, G.E. Two-Dimensional Computational Model for Wave Rotor Flow Dynamics, ASME Paper No. 96-GT-550, 1996.

[8] Hirsch, C. Numerical Computation of Internal and External Flows, Volumes I \& II, John Wiley and Sons, 1991.

[9] Roe, P.L. Characteristic-Based Schemes for the Euler Equations. Annual Review of Fluid Mechanics, Vol. 18, pp.337-365, 1986.

[10] Kentfield, J.A.C. Nonsteady, One-Dimensional, Internal, Compressible Flows. Oxford University Press, 1993.

[11] Shreeve, R.P. and Mathur A. (editors). Proceedings of the 1985 ONR/NAVAIR Wave Rotor Research and Technology Workshop, Naval Postgraduate School, Monterey, California, 1985. 